\title{
émulations
}

\section{Alexis Martig - La reconnaissance sociale et le Mouvement des Sans Terre au Brésil. En quête de dignité}

Luis Martínez Andrade

Émulations - Revue de sciences sociales

2015, « Comptes rendus critiques, En ligne »

Article disponible à l'adresse suivante

https://ojs.uclouvain.be/index.php/emulations/article/view/7093

Pour citer cet article

Luis Martínez Andrade, «Alexis Martig — La reconnaissance sociale et le Mouvement des Sans Terre au Brésil. En quête de dignité », Émulations, en ligne. Mise en ligne le 14 décembre 2015.

DOI : 10.14428/emulations.cr.013

Distribution électronique : Université catholique de Louvain (Belgique) : ojs.uclouvain.be

(C) Cet article est mis à disposition selon les termes de la Licence Creative Commons Attribution, Pas d'Utilisation Commerciale 4.0 International. http://creativecommons.org/licenses/by-nc/4.0/

Éditeur : Émulations - Revue de sciences sociales / Presses universitaires de Louvain https://ojs.uclouvain.be/index.php/emulations

ISSN électronique : $1784-5734$

UCL PRESSES

UNIVERSITAIRES

DE LOUVAIN 


\section{Alexis Martig - La reconnaissance so- ciale et le Mouvement des Sans Terre au Brésil. En quête de dignité}

Luis Martínez Andrade ${ }^{1}$

Recensé : Alexis Martig, La reconnaissance sociale et le Mouvement des Sans Terre au Brésil. En quête de dignité, Louvain-la-Neuve, Academia-L'Harmattan, 2014, 287 p.

À coup sûr, le Mouvement des Sans-Terre (MST) représente bel et bien l'un des plus importants mouvements sociaux du «Sud Global». Héritier des luttes paysannes des années 1950 (Ligas Camponesas), du travail des syndicats, des Communautés ecclésiales de base (CEB) et de la Commission pastorale de la terre (CPT), le Mouvement des Sans Terre aspire à transformer radicalement la formation sociale hégémonique, c'està-dire la société capitaliste. Ainsi, l'intérêt pour ce mouvement s'explique non seulement par les conflits socio-économiques qui sont en jeu mais aussi par sa portée politico-culturelle. Le livre d'Alexis Martig, docteur en anthropologie de l'Université Lyon 2, offre une précieuse contribution à l'étude des aspects éthiques de ce mouvement.

À partir d'une approche axée sur les questions de reconnaissance sociale, l'auteur approche la dignité comme un objet de recherche à même d'intéresser l'anthropologie. En s'appuyant sur les apports de philosophes et d'historiens tels qu'Axel Honneth, Gayatri Spivak, Avishai Margalit, Nancy Fraser, Gilberto Freyre, Caio Prado Júnior ou José de Souza Martins, Martig mobilise et articule de nombreux concepts (tels que le "principe d'égalité universelle», la "parité de participation », l'« humiliation institutionnelle » ou la « disposition de l'esprit ») et théories dans le but de présenter le cadre socio-historique des luttes paysannes au Brésil. Ainsi, il écrit que «si la question centrale et à l'origine du MST est bien la question du combat contre la pauvreté spécifique de la population des travailleurs ruraux brésiliens, avec pour solution la réforme agraire, la notion de dignité apparaît néanmoins elle aussi comme centrale dans le projet du mouvement social » (p. 51). La réforme agraire et la dimension économique de ce mouvement ne sont pas écartées par l'auteur, bien qu'il concentre son attention sur les logiques sociales inhérentes aux processus de la reconnaissance.

${ }^{1}$ Docteur en sociologie de l'École des hautes études en sciences sociales. Chercheur au CriDIS (Centre de recherches interdisciplinaires Démocratie, Institutions, Subjectivité), Université catholique de Louvain. 
Dans une démarche ethnographique, Martig se focalise sur le Setor de Cultura ${ }^{2}$ afin d'analyser aussi bien les discours que les pratiques du MST. L'anthropologie de la dignité qu'il propose a vocation à éclairer les contextes où ont lieu des luttes sociales. En dressant un aperçu socio-historique du monde rural (chapitre 2), l'auteur montre comment les populations rurales se trouvent en position de subordination dans la structure socio-économique brésilienne. La spécificité de la colonisation portugaise au Brésil, la monoculture des latifundios ${ }^{3}$, la domination personnelle et impersonnelle (la relation établie entre le propriétaire terrien et le paysan par le biais du parrainage), la concentration foncière et la consolidation d'une structure bimodale forment la toile de fond des conflits et des antagonismes dans le monde rural brésilien.

Concernant la formation du MST, Martig souligne le fait que l'exclusion des paysans du pacte politique a eu des effets néfastes pour la population rurale. À ce sujet, l'auteur relève que « la Constitution de 1824 affirmait l'exclusion politique des pauvres en restreignant leur accès au statut d'électeur ou à l'éligibilité selon des critères économiques, ceci jusqu'à la proclamation de la République en 1889» (p. 96). Loin d'en finir avec les inégalités sociales, l'abolition de l'esclavage en 1888 a reconfiguré les relations de domination, de sorte que les maîtres d'esclaves sont devenus des « seigneurs des terres ». $\mathrm{Au}$ fur et à mesure que les organisations paysannes se sont organisées vis-à-vis des grands propriétaires terriens, les conflits pour l'accès à la terre se sont intensifiés. Dans les années 1950, grâce au travail des Ligas Camponesas (Ligues paysannes), les organisations paysannes ont acquis une visibilité grandissante dans l'espace politique. Cependant, à partir du coup d'État de 1964, les organisations paysannes, populaires et de gauche, ont été violemment réprimées.

Durant les années 1970 et 1980, l'action combinée des Communautés ecclésiales de base ${ }^{4}$, des militants du Parti communiste et de la Pastorale de la Terre5 (fondée en 1975), a fortement contribué à une prise de conscience politique dans le monde rural. À cet égard, Martig souligne le fait que « La CPT (Commission Pastorale de la Terre) fut à la

\footnotetext{
${ }^{2}$ Secteur de la Culture.

${ }^{3}$ Grand domaine agricole privé, d'exploitation archaïque, utilisant une main-d'œuvre non spécialisée et fort mal rétribuée.

¿ La «Communauté Ecclésiale de Base » est la réunion de la communauté chrétienne afin de vivre le lien entre l'évangélisation, la bonne nouvelle de la libération des pauvres et des opprimés, ainsi que la pédagogie des opprimés pratiquée comme une conscientisation libératrice. Cette organisation des communautés s'est faite à la demande officielle et écrite de l'épiscopat latino-américain réuni en 1968 à Medellín (en Colombie), avec le pape Paul VI. II s'agit de l'actualisation de Vatican II en Amérique latine.

${ }^{5}$ « La Commission Pastorale de la Terre plonge ses racines dans le socialisme chrétien, paradigme théologico-politique d'une partie importante de la théologie de la libération (...). Ses membres sont essentiellement des religieux (prêtres, parfois évêques, sœurs et frères de diverses congrégations, diacre) et des laïques (théologiens, agents de pastorale, mais aussi sociologues). Dépendant directement de la Conférence nationale des évêques brésiliens, la CPT n'était pas soumise aux structures locales de l'Église » (Taleb, 2014 : 57).
} 
fois une source autonome de mobilisations paysannes, de luttes nombreuses contre les expulsions de posseiros et pour l'expropriation des fazendas, ainsi qu'une force soutenant le syndicalisme là où il était le plus combatif. Enfin, c'est dans la CPT que furent formés les principaux leaders, fondateurs et penseurs du MST » (p. 107). Ainsi, le MST a vu le jour entre la rencontre de janvier 1984 au Centre diocésain de formation de Cascavel dans l'État du Parana et le premier congrès des associations de paysans sans terre en 1985 à Curitiba. Il est intéressant de noter que le MST a toujours su conserver son autonomie bien qu'il a reçu un soutien non-négligeable de la part de l'Église et a été fortement influencé par celle-ci, étant proche de la théologie de la libération.

C'est à travers les místicas (une forme de performance basée sur l'usages de pratiques artistiques diverses et plurielles) que nous pouvons mieux saisir l'ampleur de l'influence de la théologie de la libération sur le MST (chapitre 6). Aux yeux de Martig, le rôle de la mística est d'alimenter la militance et, en conséquence, de développer le caractère subjectif des militants. À notre avis, l'élément le plus intéressant de cette section réside dans les analyses portant sur les « déclinaisons temporelles » (p. 201). Face à la temporalité vide et homogène de la modernité-capitaliste, les místicas donnent une autre tonalité aux divers temps (présent-passé-futur). Ce nouveau temps vécu mobilise le passé non comme retour mais plutôt comme détour pour projeter un horizon (l'utopie concrète chère à Ernst Bloch, 1991) qui relève d'une praxis révolutionnaire, à l'œuvre ici et maintenant. En ce sens, Martig soutient que « l'intérêt d'une participation à une mística réside dans la capacité de mise à distance de l'Histoire, permise par le temps messianique qui vient interrompre la logique linéaire et la longueur du temps constitutif du quotidien des travailleurs ruraux » (p. 207).

L'analyse des activités du Setor de Cultura du MST, à partir d'une enquête ethnographique, proposée par Martig (chapitres 4,5 et 7), montre l'esthétisation du politique et la politisation de l'esthétique, les valeurs anti-hégémoniques, la transformation subjective des travailleurs ruraux, l'importance de la conscience esthétique et l'usage du théâtre en tant qu'instrument de lutte. Or, comme l'observe l'auteur, le Setor de Cultura est un des secteurs les plus récents du MST, formé seulement en 1996 par le biais du Coletivo nacional de cultura. Il déploie son travail autour de trois axes, à savoir : (1) la qualification esthétique et politique de militants dans les langages culturels, (2) la production d'un référentiel de culture allant à l'encontre de la logique de production culturelle du capitalisme et (3) le renforcement du contact entre la population rurale et urbaine. Pour Martig, les symboles sont importants pour contribuer à la constitution d'une identité propre à ce mouvement ainsi qu'à une mémoire collective associée. À titre d'exemple, le massacre d'Eldorado dos Carajas en avril 1996 dans l'État de Para est un passage incontournable dans les représentations artistiques et politiques du MST.

Cet ouvrage contribue donc à une anthropologie du politique qui accorde une attention particulière au lien entre culture et pouvoir. La démarche ethnographique employée par l'auteur lui permet de mettre en lumière les défis et les tensions qui se trouvent dans les activités du Setor de Cultura du MST. Selon nous, l'apport de ce travail 
réside dans la mise en perspective des activités culturelles et artistiques développées par ce mouvement que la méthodologie utilisée rend possible. En outre, ce livre se plonge de façon convaincante dans l'analyse de la voie de la subjectivité (Pleyers 2010) propre au MST. Il interpellera les anthropologues, les historiens et les sociologues intéressés non seulement par l'analyse de la reconnaissance sociale mais aussi intrigués par l'histoire et le parcours de la lutte pour la terre au Brésil.

\section{Bibliographie}

BLoch E. (1991) : Le Principe Espérance (3 volumes). Paris : Gallimard.

PLEYERS G. (2010) : Alter-globalization. Becoming actors in the Global Age. Cambridge : Polity Press.

TALEB M. (2014) : L'Écologie vue du Sud. Pour un anticapitalisme éthique, culturel et spirituel. Paris : Sang de la Terre. 